\title{
Patterns of within population dispersal and mating of the fungus Microbotryum violaceum parasitising the plant Silene latifolia
}

\author{
T Giraud \\ Ecologie, Systématique et Evolution, UMR 8079 CNRS-UPS, Bâtiment 360, Université Paris-Sud, F-91405 Orsay cedex, France
}

This study explores the patterns of dispersal and mating of the anther smut Microbotryum violaceum, a model species in genetics and evolutionary biology. A French metapopulation of the fungus collected from its caryophyllaceous host Silene latifolia was analysed using microsatellites. The genetic diversity was low, populations were strongly differentiated, and there was no pattern of isolation by distance among populations. There was a strong deficit in heterozygotes, confirming the high self-fertilisation rates suggested by previous studies. Within populations there was a strong pattern of isolation by distance, with identical genotypes being highly clustered. This indicates that fungal spores are dispersed mostly between adjacent plants, and such local dispersal is important for understanding the dynamics and evolution of this disease. Local clusters of identical heterozygous genotypes did not contain significantly fewer individuals than did clusters of homozygous genotypes. As selfing between products of independent meiotic events (intertetrad selfing) rapidly reduces heterozygosity, this suggests that intratetrad matings are frequent, which helps to explain the puzzling maintenance of a sex-ratio distortion in $M$. violaceum. Heredity (2004) 93, 559-565. doi:10.1038/sj.hdy.6800554 Published online 4 August 2004

Keywords: Ustilago violacea; basidiomycete; isolation by distance; spore dispersal; selfing; metapopulation; parasite

\section{Introduction}

Knowledge of modes of dispersal and mating of parasites is essential for investigating the transmission and evolution of diseases. Parasite dispersal is one of the key factors affecting the dynamics and coevolution of hostparasite interactions (Thompson, 1994). The degree of parasite dispersal among populations has indeed had an influence on the pattern of local adaptation (Gandon and Van Zandt, 1998), the evolution of host resistance (Barrett, 1980), and the very coexistence of hosts and pathogens (Thrall et al, 1993; Thrall and Antonovics, 1995). Pattern of within-population dispersal of pathogens may affect the evolution of their virulence: in a population with local dispersal, individuals tend to be aggregated with their relatives, and one expects a decrease in virulence by kin selection (Van Baalen and Rand, 1998). However, despite its importance, few empirical studies have examined parasite dispersal. Those that have usually reported strong isolation between populations of parasites (Blouin et al, 1995; Delmotte et al, 1999; Garcia et al, 2002; Halliday and Carter, 2003; McCoy et al, 2003). Within-population dispersal is even more rarely investigated in parasites. The few studies on the subject have not detected patterns of isolation by distance (Jerome and Ford, 2002; McCoy, 2003), indicating free dispersal within populations.

Knowledge of modes of mating is also important for understanding the evolution of parasites. Outcrossing

Correspondence: T Giraud, Ecologie, Systématique et Evolution, UMR 8079 CNRS-UPS, Bâtiment 360, Université Paris-Sud, F-91405 Orsay cedex, France. E-mail: Tatiana.Giraud@ese.u-psud.fr

Received 15 October 2003; accepted 9 June 2004; published online 4 August 2004 promotes gene exchange and can hence speed up the spread of new beneficial mutations, which is critical in the context of the arms race between hosts and pathogens. Moreover, outcrossing permits avoidance of inbreeding depression that is known to occur in some parasites (Christen et al, 2002). However, selfing provides a gene transmission advantage (Charlesworth, 1980), as well as an insurance of reproduction for organisms having a low probability of finding a mating partner, such as parasites that frequently end up alone in a host individual. The relative rate of selfing versus outcrossing is therefore especially interesting in parasites, but has been studied to date almost exclusively in host species.

This study therefore investigated the population structure of the anther smut Microbotryum violaceum (formerly Ustilago violacea) in order to determine its patterns of dispersal and mating. This fungus is a basidiomycete obligate parasite of many Caryophyllaceae. Infected plants produce teliospores instead of pollen. In dioecious plant species, infected females develop spore-bearing anthers. Spores are transported from diseased to healthy plants by insects that usually serve as pollinators for the host plant (Roche et al, 1995; Shykoff and Bucheli, 1995), and so has the dynamics of a veneral disease (Antonovics et al, 1995). The disease sterilises but does not kill its host plant. Dispersing spores are diploid and, once deposited on a host plant, they undergo meiosis and give rise to four haploid cells (two of mating-type A1 and two of mating-type A2). Each of these cells buds off yeast-like sporidia and new infectious dikaryons are produced by conjugation of two cells of opposite mating types. $M$. violaceum has been studied as an experimental model species in genetics (reviewed in Garber and Ruddat, 2002), host-race 
differentiation (eg Biere and Honders, 1996; Shykoff et al, 1999; Bucheli et al, 2000), and sex-ratio distortion (Kaltz and Shykoff, 1997; Oudemans et al, 1998; Hood and Antonovics, 2000; Thomas et al, 2003). M. violaceum has also been the basis for theoretical models on transmission dynamics (eg Thrall et al, 1993; Antonovics et al, 1994, 1995; Thrall and Antonovics, 1995).

To date, the within-population transmission dynamics of this fungal pathogen remains primarily the subject of theoretical models, because genetic markers lacked the power to discriminate among genotypes within populations. Patterns of interhost races and interpopulation differentiation have been explored with molecular markers (Delmotte et al, 1999; Shykoff et al, 1999; Bucheli et al, 2000, 2001), but within-population data are still lacking. Detailed knowledge of the within-population genetic structure of $M$. violaceum is, however, essential both to complete the understanding of the modes of dispersal and evolution of this disease and to obtaining realistic values for important life history trait parameters to be integrated in theoretical models.

Mating parameters, essentially self-fertilisation rates, are also important for understanding the dynamics and evolution of the disease. Further, the mating system of $M$. violaceum exhibits several intriguing peculiarities, with important evolutionary consequences. $M$. violaceum dikaryons can be produced by selfing in two distinct ways: through intratetrad (= intrameiotic) or through intertetrad matings (Hood and Antonovics, 2000), and the relative importance of outcrossing, intertetrad selfing and intratetrad selfing in natural populations is not fully known. As intratetrad matings maintain more heterozygosity than intertetrad matings (Kaltz and Shykoff, 1997; Hood and Antonovics, 2000), the occurrence of intratetrad selfing can reduce the deleterious effect of inbreeding. From an experimental point of view, this also renders difficult the inference of outcrossing rate from the knowledge of heterozygosity, as can typically be done in population genetics studies of other species.

Knowledge of the mating patterns is also needed to understand the maintenance of the puzzling sex-ratio distortion in $M$. violaceum. Some $M$. violaceum strains produce diploid spores all giving rise to sporidia of only one mating type, although all diploid spores are heterozygous at the mating-type locus (Kaltz and Shykoff, 1997; Oudemans et al, 1998; Hood and Antonovics, 2000; Thomas et al, 2003). Such individual sex-ratio distortion has been explained by haplolethal alleles linked to one mating type (Oudemans et al, 1998). Strains with a biased sex ratio still produce four cells following meiosis, two of each mating type, but sporidia carrying the haplolethal allele quickly die after a few mitotic divisions (Hood and Antonovics, 2000). Teliospores of a strain with a biased sex ratio are still able to produce an infectious dikaryon, but only by early intratetrad conjugation (Oudemans et al, 1998; Hood and Antonovics, 2000). The frequency of intratetrad matings under natural conditions is therefore important for understanding the maintenance of high frequencies of haplolethal alleles in natural populations.

Here, I investigate the genetic population structure of $M$. violaceum in a natural metapopulation of the plant Silene latifolia, in order to examine within-population patterns of dispersal and mating. The following ques- tions were more specifically addressed: (1) Are populations founded by a limited number of fungal strains or by numerous independent migrants? (2) Does the fungus disperse within population preferentially between nearby plants? (3) Is it possible to infer from the genetic population structure the selfing rate and the frequency of intratetrad matings that occur during selfing?

\section{Material and methods}

\section{Teliospore collection and populations}

Teliospores of $M$. violaceum were collected in May and June 2001 from roadside populations of S. latifolia in a $60 \times 30 \mathrm{~km}^{2}$ area of the Essonne region, $20 \mathrm{~km}$ south-west of Paris, France. The area of collection spanned a plateau and two smooth valleys. S. latifolia populations were easy to identify, because plants were clustered, and the different patches were distinct from each other. Almost all examined populations of $S$. latifolia were infected by $M$. violaceum, with a prevalence of $0.42 \pm 0.26$ (mean $\pm S E$, $n=60$; Thomas et al, 2003). Populations were collected on different roads, or far apart, in order to cover a large area. Populations were therefore not linearly arranged, nor were all populations from the area sampled. In all, 60 populations were sampled, for which the number of infected plants and the prevalence were recorded. These $M$. violaceum populations had previously been surveyed for sex-ratio bias (Thomas et al, 2003). A random subset of 25 of these populations was genotyped in this study (Table 1 ). The mean \pm SE of the crosscountry distances between these populations was $24.1 \pm 13.1 \mathrm{~km}$.

Table 1 Characteristics of the populations of S. latifolia infected by $M$. violaceum analysed in this study: disease prevalence (percentage of infected plants), total number of infected plants (= total number of collected fungal individuals) and number of genotyped fungal individuals

\begin{tabular}{lccc}
\hline Population & $\begin{array}{c}\text { Disease } \\
\text { prevalence } \\
(\%)\end{array}$ & $\begin{array}{c}\text { Total number } \\
\text { of infected } \\
\text { plants }\end{array}$ & $\begin{array}{c}\text { Number of genotyped } \\
\text { fungal individuals }\end{array}$ \\
\hline 28 & 50 & 49 & 46 \\
31 & 30 & 26 & 22 \\
32 & 30 & 19 & 18 \\
33 & 20 & 82 & 79 \\
37 & 60 & 79 & 78 \\
47 & 85 & 62 & 43 \\
48 & 80 & 150 & 145 \\
49 & 60 & 26 & 24 \\
50 & 10 & 7 & 7 \\
52 & 50 & 22 & 22 \\
55 & 70 & 99 & 45 \\
56 & 70 & 42 & 21 \\
58 & 60 & 93 & 23 \\
A & 55 & 24 & 18 \\
C & 45 & 11 & 8 \\
D & 15 & 20 & 13 \\
E & 60 & 18 & 15 \\
G & 40 & 36 & 5 \\
M & 5 & 5 & 13 \\
N & 70 & 16 & 4 \\
O & 75 & 4 & 5 \\
P & 50 & 5 & 5 \\
R & 100 & 5 & 86 \\
T & 55 & 8 & 36 \\
V & 90 & 39 & \\
& & &
\end{tabular}


Populations were collected from roadsides, were linear and $10-300 \mathrm{~m}$ long. Adjacent plants were close to each other, mostly less than $1 \mathrm{~m}$ apart and plant density was rather homogeneous within populations. Within sampled populations, each infected plant from one side of the road was sampled, and numbered consecutively along this line. These numbers thus corresponded to their relative geographical positions. The relative positions of plants should be more relevant than the true distances, because pollinators are expected to adapt their flight to between-plants distances, particularly in populations such as these, with short and similar distances between plants (Antonovics et al, 1995; Biere and Honders, 1998; Bucheli and Shykoff, 1999). The density of plants can affect the frequency of plant visits by pollinators, but this factor was likely to have little influence here because of rather homogeneous withinpopulation densities.

Plants may rarely be infected by multiple fungal strains, but single flowers always contain a single fungal strain (Day, 1980; Hood, 2003). Therefore, a single unopened flower bud from each infected plant was collected individually into a 1.5 or $2 \mathrm{ml}$ eppendorf tube filled with silica gel and then stored at room temperature until DNA extraction. Each bud was considered as a 'fungal individual'.

\section{Genotyping}

For each of the 807 fungal strains, DNA was extracted from one anther full of spores using the Chelex (Biorad) protocol described in Bucheli et al (2001). All the 49 microsatellite loci developed for $M$. violaceum (Bucheli et al, 1998; Giraud et al, 2002) were tested for amplification on five strains, and for polymorphism on a subset of 180 strains from 12 populations. The whole collection of 807 fungal strains was then genotyped using the only two loci that were polymorphic in the subset sample, L17 and L18 (Bucheli et al, 1998). PCR amplifications were performed using a PTC 100 thermal cycler (MJ Research), with 35 cycles of $94^{\circ} \mathrm{C}$ for $30 \mathrm{~s}, 55^{\circ} \mathrm{C}$ for $30 \mathrm{~s}$, and $72^{\circ} \mathrm{C}$ for $30 \mathrm{~s}$ (the annealing temperature was adapted for each locus). Each reaction $(10 \mu \mathrm{l})$ contained $1 \mu \mathrm{l}$ of $10 \times$ reaction buffer $(50 \mathrm{mM} \mathrm{KCl}, 0.1 \%$ Triton $X-100,10 \mathrm{mM}$ Tris- $\mathrm{HCl}, \mathrm{pH} 9.0), 10 \mu \mathrm{M}$ of dNTP, $0.2 \mu \mathrm{g} / \mu \mathrm{lBSA}, 1.5 \mathrm{mM}$ $\mathrm{MgCl}_{2}, 2.5 \mathrm{pmol}$ of each primer (the forward primer being labelled with fluorescence), $0.5 \%$ o polyoxyethylene ether W-1, 0.25 U of Taq DNA polymerase (Promega), and approximately $10 \mathrm{ng}$ of sample DNA. PCR products were separated in $8 \%$ polyacrylamide gels and visualised by scanning the gel with a FMBIO II scanner (Hitachi Genetic Systems).

\section{Statistical analyses}

The correlation between the number of alleles at the most diverse locus and the number of genotyped fungal individuals was tested using JMP software (SAS Institute Inc., SAS Campus Drive, Cary, NC, USA). Other factors possibly influencing the number of alleles per population could not be tested because data had not been recorded. Distances from the closest neighbouring diseased populations, for instance, were not known because not all populations from the area were sampled. Total plant population sizes (including healthy and diseased plants) could not be derived from prevalence because only one side of the road was sampled. Some populations grew on one side and others on both sides and this information had not been recorded.

Statistical analyses on population structure were performed using the updated version 3.2a of the GENEPOP software (Raymond and Rousset, 1995), available free at http://www.cefe.cnrs-mop.fr/. Wherever necessary, levels of significance of multiple tests were corrected by the sequential Bonferroni's method described by Rice (1989).

Global linkage disequilibrium between the two polymorphic loci L17 and L18 was tested using a Fisher's exact test for each locus and computing (Markov chain method) an unbiased estimate of the exact probability. The null hypothesis $\mathrm{H}_{0}$ of this test is: 'Genotypes at one locus are independent from genotypes at the other locus'.

Deviations from Hardy-Weinberg expectations were tested for each locus and each population, and a Fisher's combined probability test was performed to assess the overall significance of multiple tests across all loci and all populations.

Genic differentiation between each pair of populations was assessed using an unbiased estimate of the $P$-value of the probability test (or Fisher's exact test), as described by Raymond and Rousset (1995). The null hypothesis of this test is $\mathrm{H}_{0}$ : 'the allelic distribution does not differ across populations'. Genotypic differentiation among populations was assessed using an unbiased estimate of the $P$-value of a $\log$-likelihood (G)-based exact test. The null hypothesis $\mathrm{H}_{0}$ of this test is: the genotypic distribution does not differ across populations'.

Isolation by distance was tested by computing a Mantel test between the two half-matrices of pairwise genetic distances and pairwise geographical distances. For interpopulation tests, $\mathrm{F}_{\mathrm{ST}} /\left(1-\mathrm{F}_{\mathrm{ST}}\right)$ values were used as genetic distances and true geographical (crosscountry) distances were used, as estimated in centimetres from a road map. For intrapopulation tests, genetic distances were estimated as described in Rousset (2000), and relative distances (rank order) between infected plants were used as geographical distances. Isolation by distance was tested only within the seven populations with more than six different genotypes. Both GENEPOP (Raymond and Rousset, 1995) and SPAGeDi (Hardy and Vekemans, 2002) were used to compute the Mantel tests because they use different coefficients to test the correlation between genetic and geographic distances (rank-correlation coefficient in GENEPOP versus Pearson's correlation coefficient in SPAGeDi). Similar results were obtained with the two packages.

To test whether the clusters of identical heterozygous genotypes were smaller, that is, contained fewer individuals than the clusters of identical homozygous genotypes, I took the frequencies of each size of cluster (1, 2, 3, etc individuals) of the distribution of homozygous genotypes as the expected frequencies for the distribution of heterozygous genotypes. I then derived the number of observations expected for each size of cluster in the distribution of the heterozygous genotypes. As some expected values were inferior to 5 for the large clusters, I pooled the classes of 3 and 4 individuals and the classes of 5 or more individuals. I then compared these expected values to the observed distribution of heterozygous genotypes using a $\chi^{2}$ test. 


\section{Results}

Genetic diversity

Of the five microsatellite markers developed by Bucheli et al (1998) on a S. latifolia strain, two were polymorphic, L17 and L18. Of the 44 microsatellite markers developed by Giraud et al (2002) on other host races of M. violaceum (on strains from the plants Gypsophila repens, Dianthus sylvestris and S. vulgaris), only $25(57 \%)$ crossamplified in strains from S. latifolia, and none was polymorphic. The 25 populations from Essonne were therefore genotyped with the two markers L17 and L18. Eight alleles were detected for L17 and five alleles for L18.

To investigate whether populations were founded by several independent migrants, I analysed the number of alleles per locus because it was usually not possible to assess whether different genotypes were independent migrants or were derived from the same parental genotypes through selfing or outcrossing. I analysed the number of alleles at the most diverse locus for each population because this variable best indicates the minimal number of independent migrants that arrived in each population. The mean $\pm \mathrm{SE}$ of the number of alleles at the most diverse locus was $3.4 \pm 0.3$ and most populations, even the smallest, had two or more alleles at their most diverse locus. The number of alleles at the most diverse locus increased with the number of genotyped fungal individuals (Figure 1), which corresponds well to the total number of infected plants (minus the few from which complete genotypes were not obtained) and the correlation was highly significant $(r=0.65, P=0.0003)$.

\section{Hardy-Weinberg proportions}

No linkage disequilibrium was found between the L17 and L18 loci. Deviations from Hardy-Weinberg expectations were significant for 21 of the 25 the populations. Fisher's combined probability test across loci and populations was highly significant $(P<0.00001)$. F IS values were very high in all populations and highly significant across populations and loci $\left(\mathrm{F}_{\mathrm{IS}}\right.$ across populations was $0.89 \pm 0.02$ for L17 and $0.77 \pm 0.06$ for L18). The ratio of observed relative to expected heterozygotes was $0.148 \pm 0.173$ over all populations.

\section{Differentiation among populations}

Populations were strongly differentiated. The genic differentiation tests were highly significant for all pairs of populations and both loci $(P<0.001)$. The allelic distribution was clearly different among populations (data not shown), leading to a highly significant genotypic differentiation $(P<0.0001)$. No significant isolation by distance was found among populations.

\section{Isolation by distance within populations}

Isolation by distance was significant within each of the seven populations tested (Mantel tests, $P<0.01$ ). This isolation by distance was mostly due to adjacent plants often being infected by identical genotypes, as illustrated in Figure 2 for the six populations with the highest

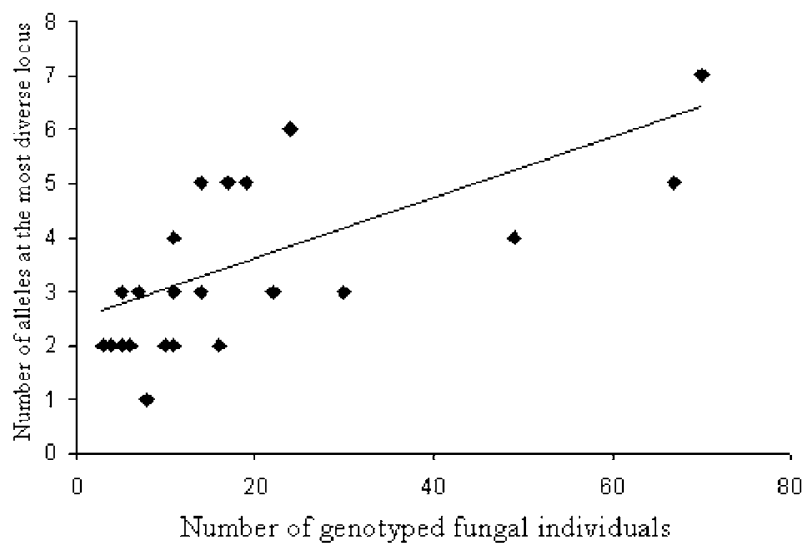

Figure 1 Number of alleles at the most diverse locus plotted against the number of fungal individuals genotyped in the population, that is, the total number of infected plants minus the few from which complete genotypes could not be obtained, for the 25 populations analysed in this study. The grey line represents the function that best fit the data.

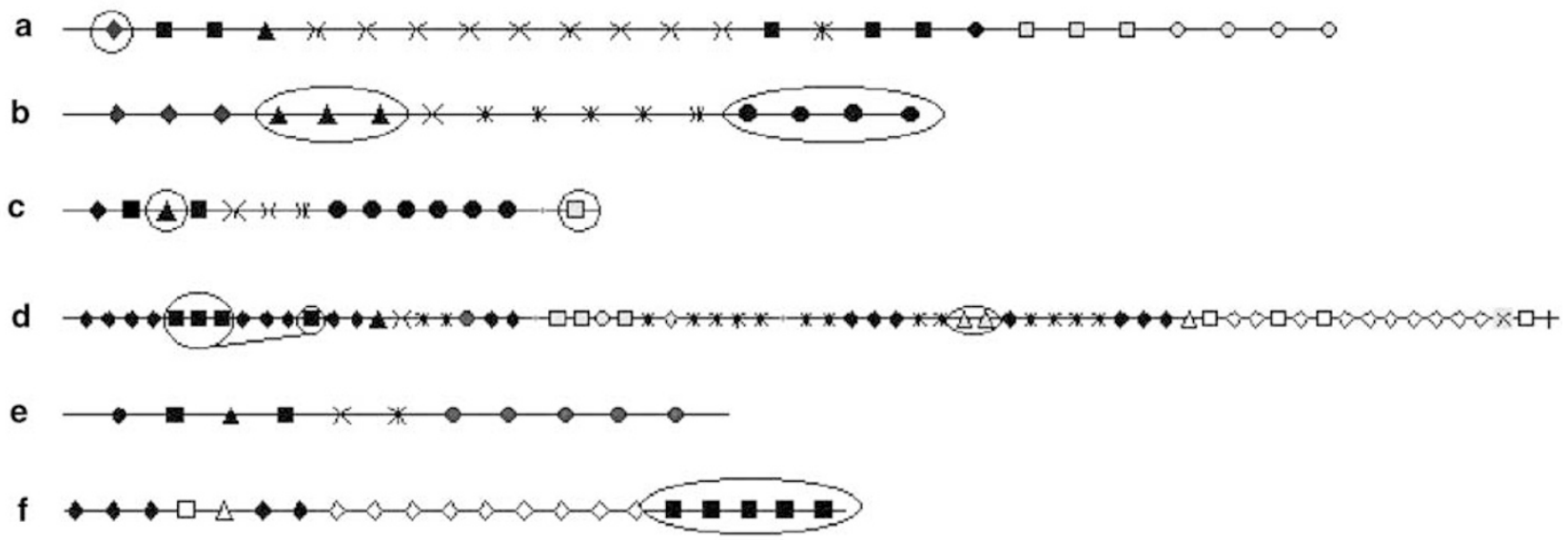

Figure 2 Patterns of within-population genotype clustering in six populations. Each different genotype is figured as a different symbol, and the axis represents the relative positions of infected plants for which the fungal genotype was obtained. Heterozygous genotypes are circled. Same symbols across populations do not represent identical genotypes. (a) population 55; (b) population 31; (c) population G; (d) population A; (e) population 33; and (f) population 49 . 


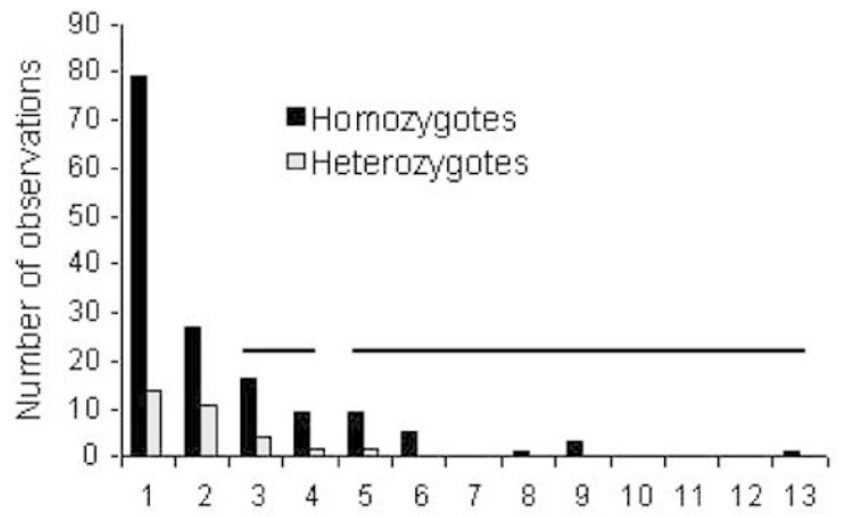

Number of individuals in a spatial cluster

Figure 3 Compared distributions of the size of the spatial clusters for homozygous and heterozygous genotypes. The thick lines signal the classes that were pooled for the statistical analysis.

numbers of different genotypes. Clustering of several individuals with identical genotypes was observed even for heterozygous fungal individuals (circled in Figure 2). Clusters of heterozygous genotypes were not significantly smaller than clusters of homozygous genotypes (Figure 3; $\chi^{2}=6.51,3 \mathrm{df}, 0.1>P>0.05$ ). However, the test was nearly significant and large clusters of identical heterozygous genotypes seemed to be missing in the distribution (Figure 3).

\section{Discussion}

Only $57 \%$ of the microsatellite markers developed on other host races crossamplified in the strains from $S$. latifolia. This is in agreement with previous studies showing strong differentiation between host races of $M$. violaceum, which might be considered as different species (Biere and Honders, 1996; Shykoff et al, 1999; Bucheli et al, 2000). Among amplifiable microsatellites, only $6.7 \%$ were polymorphic. This low genetic diversity is consistent with other work assessing variability in M. violaceum for virulence (Alexander and Antonovics, 1993), isozymes (Antonovics et al, 1996), or microsatellites (Delmotte et al, 1999; Shykoff et al, 1999; Bucheli et al, 2001). The low genetic diversity of $M$. violaceum, lower than the genetic diversity reported in other parasites (eg Blouin et al, 1995; McCoy et al, 2003), can be attributed to small effective sizes and to frequent founder events. Roadside populations of S. latifolia are indeed often disturbed and plant and fungal populations regularly go extinct (Antonovics et al, 1994). The two loci used in this study were, however, polymorphic enough to detect interesting patterns in the population structure of $M$. violaceum.

Strong interpopulation differentiation was found, as reported in previous studies on $M$. violaceum (Delmotte et al, 1999; Bucheli et al, 2001) and in several examples from other parasites (Blouin et al, 1995; Garcia et al, 2002; Halliday and Carter, 2003; McCoy et al, 2003). Strong interpopulation differentiation suggests low migration rates, which is unsurprising for parasites with restricted dispersal. Numbers of alleles at the most diverse locus found in the present study, however, suggest that a given population of $M$. violaceum is not founded by a single strain, which then spreads to healthy plants. Given the large excess of homozygotes in this fungus, the fact that almost all populations had at least two alleles, and often more at their most diverse locus, indeed indicates that independent migrant strains must regularly arrive, possibly from different source populations. This result indicates that populations of $M$. violaceum contain genetic variation, through gene immigration and gene exchange, that could give them the potential for local adaptation, although previous studies have shown that this potential is less important for the fungus than for its host S. latifolia (Kaltz et al, 1999). There was a significant positive correlation between the number of alleles at the most diverse locus and the number of genotyped fungal individuals, corresponding to the number of infected plants in each population (minus the few samples that could not be genotyped). One explanation for such a relationship is that large fungal populations represent older populations that have accumulated more immigrants. However other factors which, unfortunately, are not known in this study, are also likely to influence the number of independent fungal migrants per population. Total plant population size (including all healthy plants), distance to neighbouring populations, population densities and topography can, for instance, play on attractivity of a given plant population towards pollinisators and hence on the probability that this population receives more immigrant spores.

The high deficits in heterozygotes found in this study (only 15\% of the expected heterozygotes were found on average) and others (Delmotte et al, 1999; Bucheli et al, 2001) suggest high rates of inbreeding. Further, as $M$. violaceum self frequently through intratetrad matings (Hood and Antonovics, 2000), which retain more heterozygosity than intertetrad selfing, inbreeding could be even higher than that inferred from heterozygosity levels. As the proportion of intratetrad matings is not known, and neither are the genomic positions of the microsatellite loci, it is impossible to derive a precise selfing rate from the heterozygote deficit, but it is likely to be the predominant mode of reproduction. It was then surprising to find no linkage disequilibrium between the two microsatellite loci, but this could be due to low statistical power because genetic diversity was low. The presence of different genotypes in the same population demonstrates that there are potential partners for outcrossing. High selfing rates might, therefore, indicate a preference. Some data indeed indicate that a preference for selfing versus outcrossing in M. violaceum. Artificial inoculations experiments with combinations of teliospores bearing colony colour markers (Baird and Garber, 1979) detected an outcrossing rate of only $6.6 \%$. Kaltz and Shykoff (1999) found that mixtures of sporidia of opposite mating types conjugate more readily in selfing than outcrossing combinations.

The high selfing rates in $M$. violaceum could, alternately, be due to very local spore dispersal (see below), restricting actual outcrossing opportunities. Few studies have examined mating systems in other parasites but those that exist also found high selfing rates (Trouvé et al, 1996; Ariey et al, 1999). This may be related to a general lack of opportunities for parasites to encounter unrelated mating patners within their individual host. Such restricted gene exchange may have evolutionary consequences in the arms race context, slowing down adaptation of parasites and thus counterbalancing the 
potential advantage when they have shorter generation times.

The lack of isolation by distance among populations found in this study is in agreement with another study on M. violaceum from S. latifolia (Delmotte et al, 1999), and indicates that the probability of dispersal of the fungus from one population to another is not dependent on the distance between the two populations. Metapopulations of $M$. violaceum thus appear to function more as island models than as stepping-stone models, although those of the host plant S. latifolia appear more consistent with a stepping-stone model (Delmotte et al, 1999). This discrepancy may be due to the fact that plants disperse by seeds in addition to their pollen being transported by pollinators, as are spores of the fungus. Seed dispersal is indeed likely to be local and therefore to yield an isolation by distance pattern. The lack of isolation by distance among $M$. violaceum populations suggests that pollinators, when moving from one patch to another, do not always fly to closest patches. Such behaviour may be mediated by preferential attraction towards large or dense populations rather than towards close populations, or by landscape obstacles to their vision, such as woods, buildings or hills.

In contrast to among-population patterns, a strong pattern of isolation by distance was found within populations, suggesting that spores are dispersed by pollinators preferentially between nearby plants. This was suggested by experimental studies (Alexander and Antonovics, 1988; Roche et al, 1995) showing that spore deposition on healthy plants was related to the number and proportion of diseased flowers close by. Alternatively, clusters of fungal genotypes may reflect the underlying structure of host resistance: a given genotype may most easily spread to nearby plants that are likely to be genetically similar to the source plant. This hypothesis, however, assumes that there is some variability in the virulence of $M$. violaceum within populations, for which evidence is equivocal (Alexander and Antonovics, 1993; but see Kaltz and Shykoff 2002). Population viscosity and associated isolation by distance is not a general feature of parasites (Jerome and Ford, 2002; McCoy, 2003), so this observation in $M$. violaceum may be due either to the peculiar behaviour of pollinators carrying spores of this fungus or to the clustering of similar genotypes in this host plant due to passive seed dispersal. Such patterns may be atypical in animal hosts or animal dispersed hosts.

Further, more can be deduced from the existence of clusters of heterozygous genotypes than merely local dispersal. Since the diploid teliospore undergoes meiosis and conjugation at the site of each new infection, clusters of heterozygous genotypes require several selfing events that maintain heterozygosity in the progeny. The clusters of heterozygous genotypes did not contain significantly fewer individuals than the clusters of homozygous genotypes, suggesting that heterozygosity was transmitted during selfing as often as homozygosity. As normal, intertetrad selfing reduces the level of heterozygosity more rapidly than does intratetrad mating, this finding suggests that intratetrad matings are predominant. This helps to explain how the deleterious alleles responsible for sex-ratio distortion, which can only be transmitted to the next generation through such peculiar selfing (Hood and Antonovics, 2000), can persist at high frequencies in natural populations of M. violaceum (Kaltz and Shykoff, 1997; Oudemans et al, 1998; Hood and Antonovics, 2000; Thomas et al, 2003). There are, however, some indications that intertetrad selfing must also occur regularly: (i) high deficits in heterozygotes are found at the level of the population, suggesting that heterozygosity is lost over the long term and (ii) there was a higher frequency of large clusters of homozygote genotypes (albeit not quite significantly different from heterozygotes).

In conclusion, in agreement with previous studies, I find low genetic diversity, high levels of self-fertilisation and strong interpopulation differentiation in a natural $M$. violaceum metapopulation on S. latifolia. More importantly, at the within-population level, several independent genotypes coexist and there is a strong viscosity of the fungal population that will be important for models aiming at understanding the evolution of this sexually transmitted disease (Van Baalen and Rand, 1998). M. violaceum is thus a highly selfing parasite dispersing locally and it is therefore not surprising that it was found to be locally maladapted (Kaltz et al, 1999).

\section{Acknowledgements}

I thank Anne Thomas, Elodie Vercken and Leila Dolmate for their help with teliospores collection, and Camille Roux, Dominique Vautrin and Michel Solignac for their help with genotyping. I am grateful to Jacqui A Shykoff, Elisabeth Fournier, Michel Solignac and three anonymous reviewers for comments on a previous version of the manuscript. This work was supported by the French Bureau des Ressources Génétiques (Grant 2001-2003).

\section{References}

Alexander HM, Antonovics J (1988). Disease spread and population dynamics of anther-smut fungus of Silene alba caused by the fungus Ustilago violacea. J Ecol 76: 91-104.

Alexander HM, Antonovics J (1993). Genotypic variation in plant disease resistance - physiological resistance in relation to field disease transmission. J Ecol 81: 325-333.

Antonovics J, Iwasa Y, Hassel MP (1995). A generalized model of parasitoid, veneral, and vector-based transmission processes. Am Nat 145: 661-675.

Antonovics J, Stratton D, Thrall PH, Jarosz AM (1996). An anther-smut disease (Ustilago violacea) of fire-pink (Silene virginica): its biology and relationship to the anther-smut disease of white campion (Silene alba). Am Midl Nat 135: 130-143.

Antonovics J, Thrall PH, Jarosz AM, Stratton D (1994). Ecological genetics of metapopulations: the Silene-Ustilago plant-pathogen system. In: Real LA (ed) Ecological Genetics. Princeton University Press: Princeton, NJ. pp 146-170.

Ariey F, Chalvet W, Hommel D, Peneau C, Hulin A, MercereauPuijalon O et al (1999). Plasmodium falciparum parasites in French Guiana: limited genetic diversity and high selfing rate. Am J Trop Med Hyg 61: 978-985.

Baird ML, Garber ED (1979). Genetics of Ustilago violacea. V. Outcrossing and selfing in teliospore inocula. Bot Gaz 140: 89-93.

Barrett JA (1980). Pathogen evolution in multilines and variety mixtures. J. Plant Dis Prot 87: 383-396.

Biere A, Honders SC (1996). Host adaptation in the anther smut fungus Ustilago violacea (Microbotryum violaceum): infection success, spore production and alteration of floral traits on two host species and their F1-hybrid. Oecologia 107: 307-320. 
Biere A, Honders SC (1998). Anther smut disease transmission in Silene latifolia and Silene dioica: impact of host traits, disease frequency, and host density. Int J Plant Sci 159: 228-235.

Blouin MS, A YC, Courtney CH, Dame JB (1995). Host movement and the genetic structure of populations of parasitic nematodes. Genetics 141: 1007-1014.

Bucheli E, Gautschi B, Shykoff JA (1998). Isolation and characterization of microsatellite loci in the anther smut fungus Microbotryum violaceum. Mol Ecol 7: 665-666.

Bucheli E, Gautschi B, Shykoff JA (2000). Host specific differentiation in the anther smut fungus Microbotryum violaceum as revealed by microsatellites. J Evol Biol 13: 188-198.

Bucheli E, Gautschi B, Shykoff JA (2001). Differences in population structure of the anther smut fungus Microbotryum violaceum on two closely related host species, Silene latifolia and S. dioica. Mol Ecol 10: 285-294.

Bucheli E, Shykoff JA (1999). The influence of plant spacing on density-dependent versus frequency-dependent spore transmission of the anther smut fungus Microbotryum violaceum. Oecologia 119: 55-62.

Charlesworth B (1980). The cost of sex in relation to mating system. J Theor Biol 84: 655-671.

Christen M, Kurtz J, Milinski M (2002). Outcrossing increases infection success and competitive ability: experimental evidence from a hermaphrodite parasite. Evolution 56: 2243-2251.

Day AW (1980). Competition and distribution studies of genetically marked strains of Ustilago violacea in the same host plant. Bot Gaz 141: 313-320.

Delmotte F, Bucheli E, Shykoff JA (1999). Host and parasite population structure in a natural plant-pathogen system. Heredity 82: 300-308.

Gandon S, Van Zandt PA (1998). Local adaptation and hostparasite interactions. Trends Ecol Evol 13: 126-214.

Garber ED, Ruddat M (2002). Transmission genetics of Microbotryum violaceum (Ustilago violacea): a case study. Adv Appl Microbiol 51: 107-127.

Garcia RA, Zapater JMM, Criado BG, Zabalgogeazcoa I (2002). Genetic structure of natural populations of the grass endophyte Epichloe festucae in semiarid grasslands. Mol Ecol 11: $355-364$.

Giraud T, Fournier E, Vautrin D, Solignac M, Shykoff JA (2002). Isolation of 44 polymorphic microsatellite loci in three host races of the phytopathogenic fungus Microbotryum violaceum. Mol Ecol Notes 2: 142-146.

Halliday CL, Carter DA (2003). Clonal reproduction and limited dispersal in an environmental population of Cryptococcus neoformans var. gattii isolates from Australia. J Clin Microbiol 41: 703-711.

Hardy OJ, Vekemans X (2002). SPAGeDi: a versatile computer program to analyse spatial genetic structure at the individual or population level. Mol Ecol Notes 2: 618-620.

Hood ME (2003). Dynamics of multiple infection and withinhost competition by the anther-smut pathogen. Am Nat 162: 122-133.

Hood ME, Antonovics J (2000). Intratetrad mating, heterozygosity, and the maintenance of deleterious alleles in Microbotryum violaceum (=Ustilago violacea). Heredity 85: 231-241.

Jerome CA, Ford BA (2002). The discovery of three genetic races of the dwarf mistetoe Arceuthobium americanum (Viscaceae) provides insight into the evolution of parasitic angiosperm. Mol Ecol 11: 387-405.
Kaltz O, Gandon S, Michalakis Y, Shykoff J (1999). Local maladaptation of the plant pathogen Microbotryum violaceum to its host Silene latifolia: evidence from a cross-inoculation experiment. Evolution 53: 395-407.

Kaltz O, Shykoff JA (1997). Sporidial mating-type ratios of teliospores from natural populations of the anther smut fungus Microbotryum (=Ustilago) violaceum. Int J Plant Sci 158: $575-584$.

Kaltz O, Shykoff JA (1999). Selfing propensity of the fungal pathogen Microbotryum violaceum varies across Silene latifolia host plants. J Evol Biol 12: 340-349.

Kaltz O, Shykoff J (2002). Within- and among-population variation in infectivity, latency and spore production in a host-pathogen system. J Evol Biol 15: 850-860.

McCoy KD (2003). Spatial genetic structure of the ectoparasite Ixodes uriae within breeding cliffs of its colonial seabird host. Heredity 91: 422-429.

McCoy KD, Boulinier T, Tirard C, Michalakis Y (2003). Hostdependent genetic structure of parasite populations: Differential dispersal of seabird tick host races. Evolution 57: 288-296.

Oudemans PV, Alexander HM, Antonovics J, Altizer S, Thrall $\mathrm{PH}$, Rose L (1998). The distribution of mating-type bias in natural populations of the anther-smut Ustilago violacea on Silene alba in Virginia. Mycologia 90: 372-381.

Raymond M, Rousset F (1995). GENEPOP (version 1.2): population genetics software for exact tests and ecumenicism. J Hered 86: 248-249.

Rice WR (1989). Analyzing tables of statistical tests. Evolution 43: 223-225.

Roche BM, Alexander HH, Maltby AD (1995). Dispersal and disease gradients of anther-smut infection of Silene alba at different life stages. Ecology 76: 1863-1871.

Rousset F (2000). Genetic differentiation between individuals. J Evol Biol 13: 58-62.

Shykoff JA, Bucheli E (1995). Pollinator visitation patterns, floral rewards and the probability of transmission of Microbotryum violaceum, a veneral disease plant. J Ecol 83: 189-198.

Shykoff JA, Meyhöfer A, Bucheli E (1999). Genetic isolation among host races of the anther smut fungus Microbotryum violaceum on three host plant species. Int J Plant Sci 160: 907-916.

Thomas A, Shykoff J, Jonot O, Giraud T (2003). Mating-type ratio bias in populations of the phytopathogenic fungus Microbotryum violaceum from several host species. Int J Plant Sci 164: 641-647.

Thompson JN (1994). The Coevolutionary Process, University of Chicago Press: Chicago, IL.

Thrall PH, Antonovics J (1995). Theoretical and empirical studies of metapopulations: population and genetic dynamics of the Silene - Ustilago system. Can J Bot 73: S1249-S1258.

Thrall PH, Antonovics J, Hall DH (1993). Host and pathogen coexistence in sexually transmitted and vector-borne diseases characterized by frequency-dependent disease transmission. Am Nat 142: 543-552.

Trouvé S, Renaud F, Durand P, Jourdane J (1996). Selfing and outcrossing in a parasitic hermaphrodite helminth (Trematoda, Echinostomatidae). Heredity 77: 1-8.

Van Baalen M, Rand DA (1998). The unit of selection in viscous populations and the evolution of altruism. J Theor Biol 193 631-648. 